\title{
Title Application of a multi-layer systems toxicology framework for in vitro assessment of the biological effects of liquids and corresponding aerosols
}

\author{
Nikolai V. Ivanov \\ PMI R\&D, Philip Morris \\ Products S.A., 2000 \\ Neuchâtel, Switzerland \\ nikolai.ivanov@pmi.com
}

\begin{abstract}
Description We previously proposed a systems toxicology framework for in vitro assessment of e-liquids. The framework starts with the first layer aimed at screening the potential toxicity of e-liquids, followed by the second layer aimed at investigating the toxicity-related mechanism of e-liquids, and finally the third layer aimed at evaluating the toxicity-related mechanism of the corresponding aerosols. In this work, we applied this framework to assess the impact of the e-liquid MESH Classic Tobacco and its aerosol compared with that of cigarette smoke (CS) from the $3 \mathrm{R} 4 \mathrm{~F}$ reference cigarette. The results showed that the cytotoxicity of the MESH Classic
\end{abstract}

Tobacco liquid was similar to the Base liquid but lower than 3R4F CS TPM at comparable nicotine concentrations. Relative to 3R4F CS exposure, MESH Classic Tobacco aerosol exposure did not cause tissue damage and elicited lower changes in the mRNA, microRNA, and protein markers. In the context of tobacco harm reduction strategy, the framework is suitable to assess the potential reduced impact of electronic cigarette aerosol relative to $C S$.

Keywords - Systems Toxicology, in vitro, e-liquid 